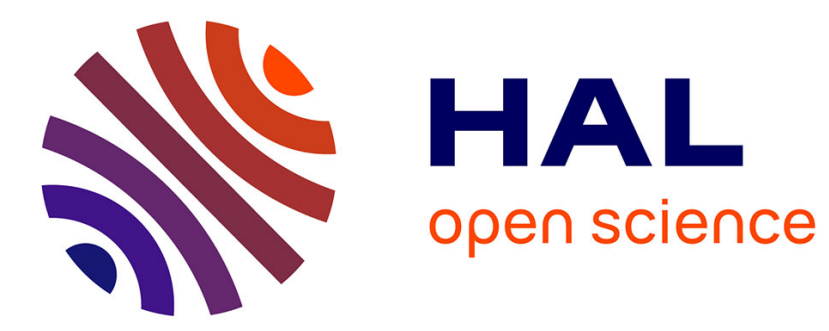

\title{
Wedge cameras for minimally invasive archaeology
}

Thomas Crespel, Adrian Travis, Patrick Reuter, Xavier Granier

\section{To cite this version:}

Thomas Crespel, Adrian Travis, Patrick Reuter, Xavier Granier. Wedge cameras for minimally invasive archaeology. Journal on Computing and Cultural Heritage, 2019, 12 (2), pp.14:1-14:13. $10.1145 / 3284425$. hal-02096396

\section{HAL Id: hal-02096396 \\ https://inria.hal.science/hal-02096396}

Submitted on 11 Apr 2019

HAL is a multi-disciplinary open access archive for the deposit and dissemination of scientific research documents, whether they are published or not. The documents may come from teaching and research institutions in France or abroad, or from public or private research centers.
L'archive ouverte pluridisciplinaire HAL, est destinée au dépôt et à la diffusion de documents scientifiques de niveau recherche, publiés ou non, émanant des établissements d'enseignement et de recherche français ou étrangers, des laboratoires publics ou privés. 


\title{
Wedge cameras for minimally invasive archaeology
}

\author{
THOMAS CRESPEL, Inria Bordeaux Sud-Ouest / LP2N / Université de Bordeaux, France \\ ADRIAN TRAVIS, Cambridge University, UK \\ PATRICK REUTER, Inria Bordeaux Sud-Ouest / Université de Bordeaux / LaBRI, France \\ XAVIER GRANIER, Institut d'Optique Graduate School / LP2N / Archéovision, France
}

\begin{abstract}
Acquiring images of archaeological artifacts is an essential step for the study and preservation of cultural heritage. In constrained environments, traditional acquisition techniques may fail or be too invasive. We present an optical device including a camera and a wedge waveguide that is optimized for imaging within confined spaces in archeology. The major idea is to redirect light by total internal reflection to circumvent the lack of room, and to compute the final image from the raw data. We tested various applications onsite during an archaeological mission in Medamoud (Egypt). Our device was able to successfully record images of the underground from slim trenches of about $15 \mathrm{~cm}$ wide, including underwater trenches, and between rocks composing a wall temple. Experts agreed that the acquired images were good enough to get useful information that cannot be obtained as easily with traditional techniques.
\end{abstract}

CCS Concepts: • Computing methodologies $\rightarrow$ Computational photography;

Additional Key Words and Phrases: camera, minimally invasive, optical device, constrained environment, digital preservation, cultural heritage

ACM Reference Format:

Thomas Crespel, Adrian Travis, Patrick Reuter, and Xavier Granier. 2019. Wedge cameras for minimally invasive archaeology. ACM J. Comput. Cult. Herit. 12, 2 (June 2019), 14 pages. https://doi.org/0000001.0000001

\section{MOTIVATION}

Imaging techniques have long played a major role in the acquisition, visualization and conservation of cultural heritage. In confined spaces, the imaging distance may not be enough for classical imaging techniques to work at their best. Indeed, taking a picture of a side of a narrow slit for example may be difficult, if not impossible, to achieve with a satisfying angle, focus or imaging area, and our work addresses this issue. The need for a minimum imaging distance might seem obvious: we all open a book in order to read it and we stretch out our arm in order to take a selfie. Archaeology is one of several disciplines like surgery and aircraft inspection where specialists seek to learn as much as they can with minimal disruption, so ensuring an imaging distance is not always possible in confined spaces.

Authors' addresses: Thomas Crespel, Inria Bordeaux Sud-Ouest / LP2N / Université de Bordeaux, 200 Avenue de la Vieille Tour, Talence, 33405, France, thomas.crespel@inria.fr; Adrian Travis, Cambridge University, Cambridge, CB2 1TL, UK, arlt1@cam.ac.uk; Patrick Reuter, Inria Bordeaux Sud-Ouest / Université de Bordeaux / LaBRI, 351 cours de la Libération, Talence, France, preuter@labri.fr; Xavier Granier, Institut d'Optique Graduate School / LP2N / Archéovision, Rue François Mitterand, Talence, France, xavier.granier@inria.fr.

Permission to make digital or hard copies of all or part of this work for personal or classroom use is granted without fee provided that copies are not made or distributed for profit or commercial advantage and that copies bear this notice and the full citation on the first page. Copyrights for components of this work owned by others than the author(s) must be honored. Abstracting with credit is permitted. To copy otherwise, or republish, to post on servers or to redistribute to lists, requires prior specific permission and/or a fee. Request permissions from permissions@acm.org.

(C) 2019 Copyright held by the owner/author(s). Publication rights licensed to Association for Computing Machinery.

XXXX-XXXX/2019/6-ART $\$ 15.00$

https://doi.org/0000001.0000001

ACM J. Comput. Cult. Herit., Vol. 12, No. 2, Article . Publication date: June 2019. 


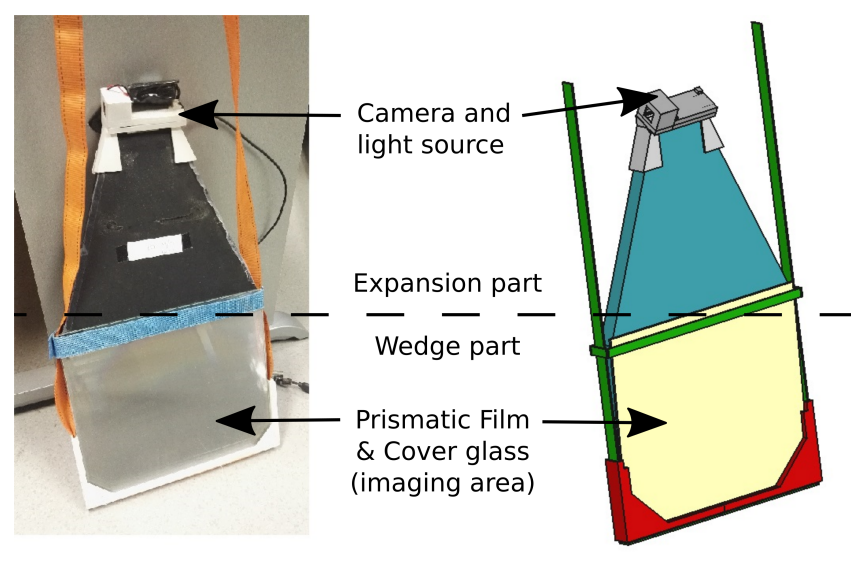

(a)

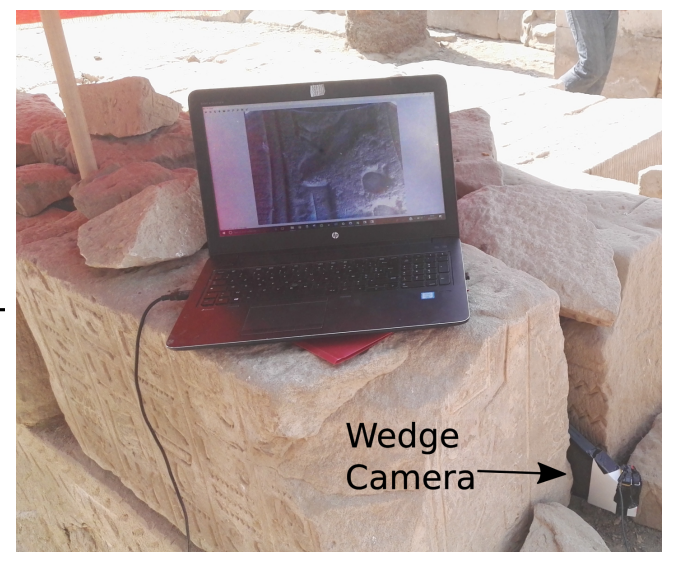

(b)

Fig. 1. (a) Annotated picture and scheme to identify the geometry and the different parts of our device. (b) The device used for inspecting rocks.

As an example, although in the archaeology of buildings, a standing structure can be studied and documented with both laser and photogrammetry [7], there are inaccessible areas that require other dedicated devices such as radar measurements [2] (notably for underground analysis) or endoscopes [1]. Endoscopes are indeed the more straighforward way to image places that are inaccessible by regular cameras, but they still require that there be a chamber, i.e. a space within which the surface under inspection can be imaged. Even if we assume that this minimum imaging distance can be achieved in some specific confined spaces, the imaging area would be very small and numerous pictures would be necessary to get a full view.

The system presented in this paper is between an endoscope and a document scanner: it can image unaccessible areas like endoscopes but larger images can be obtained without any imaging distance just like a document scanner. Unlike a document scanner, distant objects can also be imaged. Our system is based on the association of a regular camera and a wedge light-guide developed by Travis et al. [10, 12] . The whole system principle can be compared to a sort of periscope that allows imaging unaccessible areas, and we explore the utility of such a device in the specific field of archaeology.

This paper is organized as follows. In Section 2, we present the wedge guide technology, core of our imaging system for constrained environments. Then, in Section 3, we present the design of the wedge, we explain the hardware and software requirements of our prototype, and we analyze its characteristics. In Section 4, we introduce the different applications and on-site results. Finally, in Section 5, we present directions for future work, before we conclude in Section 6 .

\section{OVERVIEW OF WEDGE GUIDES}

Wedge guides were first devised for rear projection television and subsequently investigated for the human-computer interface as cameras that might allow a display to observe activity to its front [3]. To understand how they work, it helps at first to consider this part of the system in terms of projection: consider a ray of light that is shone into one end of a cuboid slab of transparent plastic. By total internal reflection, the opposing air/plastic interfaces act like mirrors so that the ray will follow the path of a 


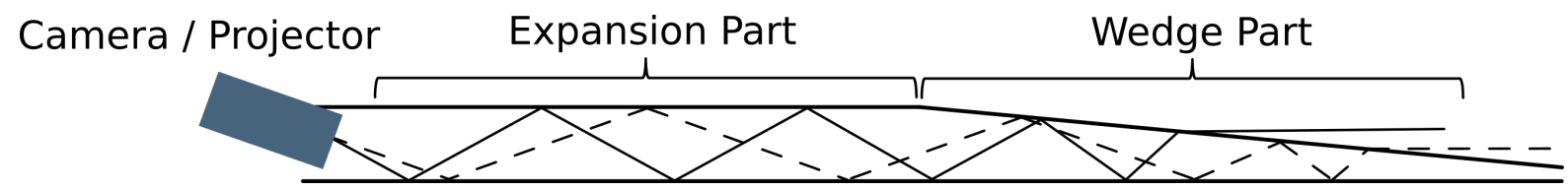

Fig. 2. Principle of the wedge. For a projector, light rays are guided in the expansion part through total internal reflection so that they can fan out horizontally, then they are extracted through the wedge part. In our work, the rays are guided from the wedge part towards the camera with the same light path.

perfect zig-zag as shown in Figure 2. Suppose, however, that we alter the slab so that one surface is slightly angled relative to the other. Each time the ray reflects off one surface, its angle relative to the other surface is reduced, like a ping-pong ball dropping into a funnel. Eventually an angle of incidence is reached, known as the critical angle, at which the ray is no longer reflected but instead passes into air.

Figure 2 shows a side view of the wedge guide that is composed of an expansion part and a wedge part. It illustrates also how the angle of injection determines the distance traveled by the ray before it leaves the guide. The plain line represents a ray that is launched so as to be near the critical angle, then the ray will need only one or two reflections within the wedge part before it reaches the critical angle, and so will not travel far. If, however, the ray is launched at a small angle to the slab axis, such as represented by the dashed line, then many reflections will be needed within the wedge part before the ray exits. This is just what happens with a video projector and a screen: the angle at which a ray leaves the projector determines how far up the screen is the point at which the ray hits it. We can therefore imagine replacing the space between a projector and screen with a wedge guide and indeed, this was one of the early applications proposed for the Optics community [10]. The image must expand to the full width of the screen as well as to its full height, and this happens simply by making the expansion part wide enough to allow rays to fan out.

A camera works much like a projector except that the direction of rays is reversed. A wedge can therefore also be used to fold up the space otherwise needed between a camera and the object that is being imaged. This means, for example, that it is possible to photograph a page in a book without opening it - one merely slides the wedge between the pages. The use of a wedge guide with a camera was already studied to image pages that are directly on the wedge surface [3] and to make the guide geometry more compact [11].

An important caveat is that as rays leave the wedge, they have only just reached the critical angle. The rays therefore emerge into air traveling almost at right angles to the surface normal. Whereas a projector launches an image that is diffused at the surface of a wedge, a camera will be most useful if it can observe images both on the surface of the wedge and far beyond. A prismatic film should be added that deflects rays parallel to the surface normal so that they can be useful, and this aspect is detailed in Section 3.1.

\section{WEDGE CAMERA DESIGN}

The wedge we use, shown in Figure 1(a), is made from polymethyl methacrylate (e.g. Plexiglas ${ }^{T M}$ ) and has an imaging area of $260 \mathrm{~mm}$ by $215 \mathrm{~mm}$, it is $455 \mathrm{~mm}$ long and $25 \mathrm{~mm}$ thick. The wedge was designed according to the principles outlined in the international patent WO03013151 [9]. The surfaces are protected by black cardboard except at the imaging surface which is covered by a prismatic film for deflecting light rays and a cover glass for protection.

A $3 \mathrm{~mm}$ glass cover slip is glued onto the wedge entrance to ensure its flatness. At the wedge entrance, there is a mount made by $3 \mathrm{D}$ printing that holds the camera and the light source. The prismatic film 
and the cover glass are held against the wedge surface with another 3D printed mount and straps. These straps are also used to bring down the wedge into trenches.

After presenting how the prisms can deflect light rays in Section 3.1, we detail the resulting equivalent camera in Section 3.2 and the illumination system in Section 3.3. The prototype was connected to a computer software to visualize and record images corrected from distortion as detailed in Section 3.4.

\subsection{Deflecting light rays}

As explained in Section 2, the rays need to be deflected in order to observe a distant object, otherwise they would simply propagate nearly parallel to the surface as shown in Figure 3(a). To this end, we propose to use a prismatic film which is an optical surface that is composed of tiny prisms elongated in one direction. A prismatic film can turn light direction in different ways.

Figure 3(b) shows that the tips of the prisms can be used as mirrors to deflect the rays to a normal direction. The problem of this method is that the reflection happens over a tiny region so diffraction tends to blur the image as shown in Figure 3(e). It is well known that when plane waves are incident on a slit, the wave that emerges will radiate slightly so that the wavefront gets bigger as it gets further from the slit. This limits how small can be the pixels of an image projected through the slit and, equivalently, limits the resolution of the picture that can be imaged via the slit. Each prism of the prismatic film acts like a slit and so the bigger the prisms, the better is the resolution of images captured distant from the

\section{Grazing angle}

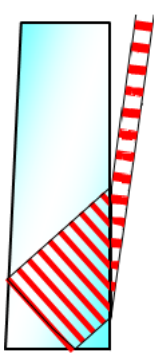

(a)

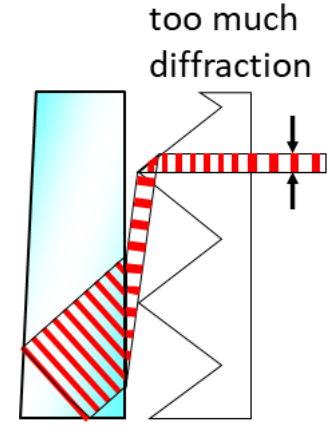

(b)

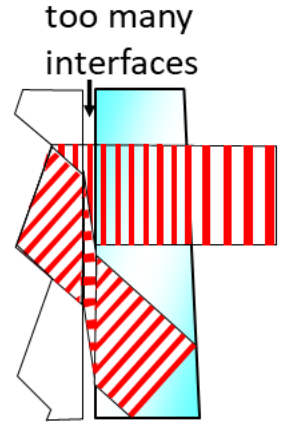

(c)

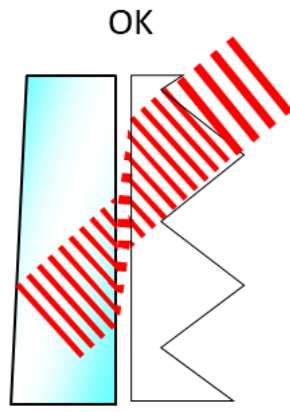

(d)

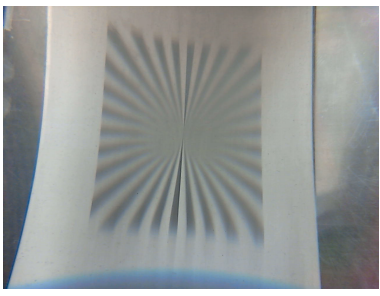

$(\mathrm{e})$

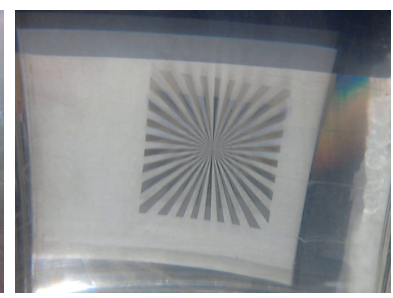

(f)

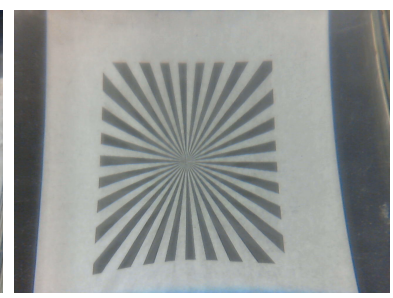

(g)

Fig. 3. (a) Without a prismatic film, distant objects cannot be imaged; (b) configuration displaying strong aperture diffraction; (c) configuration displaying too much interface reflection; (d) configuration minimizing both of the previous at the cost of tilting the angle of observation away from the normal; (e) blurred image at about $7 \mathrm{~cm}$ with configuration $\mathrm{b}$; (f) ghost image effect with configuration c; (g) sharp image at about $7 \mathrm{~cm}$ with configuration $\mathrm{d}$. 
screen. However, arrays with big prisms have big transients between each prism and this means that an object close to the surface of the film cannot be imaged in its entirety.

A possible solution might be for the prism array to act in reflection in the manner shown in Figure $3(\mathrm{c})$, but the light then has to cross many boundaries before it leaves the system and each boundary lowers the brightness and adds an unwanted reflection to the image. Anti-reflection coatings might help but would be affordable only in quantity production.

While it is important for use in the human/computer interface that rays are captured when incident perpendicular to the wedge surface, this matters much less for use in archaeology. So the prismatic film was turned over with the flat surface close to the wedge and the prisms facing the air as shown in Figure $3(\mathrm{~d})$. Light enters the material of the film at the critical angle that is approximately $45^{\circ}$ and when the light meets the prisms it travels straight on into air at the same angle. In contrast to Figure $3(\mathrm{~b})$, the aperture seen by the ray is no longer a portion of the prisms but the full prism aperture, so the diffraction effect is limited, and there are no extra surface reflections such as in Figure 3(c). In this way, the rays are deflected away from the normal but the acquired image is sharper as shown in Figure $3(\mathrm{~g})$. The prismatic film we used for this experiment is the 25FD330 from Comar Optics, with a pitch of $0.3 \mathrm{~mm}$ and a prism angle of $45^{\circ}$.

\subsection{Equivalent camera description}

Figure 4 shows the field of view (FOV) of our wedge camera system compared to an endoscope or a regular camera. The required distance between the device and the object to get a large imaging area is much longer with a regular camera or endoscope than with our wedge device. Moreover, the focal length of any endoscope lens defines a minimum distance between lens and object whereas in our system, this distance is folded up into the wedge guide and the object is in focus directly on the wedge surface.

Let us analyze the resulting FOV in more detail. Once again, for the sake of clarity, the following explanations describe rays leaving the wedge but readers must keep in mind that for acquisition, rays propagate from the object, towards the wedge guide and the camera at the end.

As explained in Section 2, the wedge width (in $x$ direction in Figure 4(c)) allows rays to fan out within the guide, and the prism array does not change this property. Rays still fan out horizontally when leaving the wedge as in a regular perspective camera. The difference is that the FOV forms a sort a trapeze because rays that leave closer to the camera fan out differently from those leaving the wedge at the end as shown in Figure 4(c). This phenomenon is similar to a keystone distortion due to off-axis projection or acquisition. Rays within the wedge are constrained in the $z$ direction and, thanks to the prismatic film, they all escape at the same angle close to $45^{\circ}$ with respect to the wedge's normal. They are still parallel to each other in the $y z$ plane as shown in Figure 4(d), so the vertical FOV is orthographic.

As a consequence, one can observe a 1D perspective: the images appear to be squeezed in $x$ direction with an increasing distance of the object from the device, whereas the vertical dimension $y$ remains the same. This is illustrated in Figure 5 where we imaged the same checkerboard at different distances, from 0 to $30 \mathrm{~cm}$ away from the device: the ratio $y: x$ changes from $1: 1$ at $0 \mathrm{~cm}$ to about $1: 2$ at $30 \mathrm{~cm}$. Note that the images get blurrier with an increasing distance due to the diffraction by the prisms. In our use context, this is not an issue since we target the acquisition in confined spaces where the objects to image are close enough.

Our imaging geometry is not standard, but has similarities with the pushbroom camera model [6]. In a pushbroom camera, a linear sensor acquires a 1D image of a scene and moves perpendicularly to the sensor direction to build a 2D image. The integrated FOV of a pushbroom camera over a finite period of 
time hence resembles the described FOV, with the difference that our device does not show time-related effects (apart from an eventual rolling shutter of the camera).

We would like to emphasize the fact that only rays incident with specific directions are guided through the wedge, so most of them are not collected. This is actually how every camera works: the lens aperture selects rays incident at a specific location with specific angles defined by the FOV and the wedge is just a way to redirect light toward the camera aperture. The depth of field, exposure time and other camera-related parameters are defined by the choice of the camera. The required specifications are a $60^{\circ}$ horizontal FOV in order to benefit from the maximum of imaging area, and an aperture that is smaller than the wedge entrance $(3 \mathrm{~mm}$ high). The camera should also have either a fixed focus with a large depth of field or an autofocus able to focus on the wedge surface and beyond. For the results

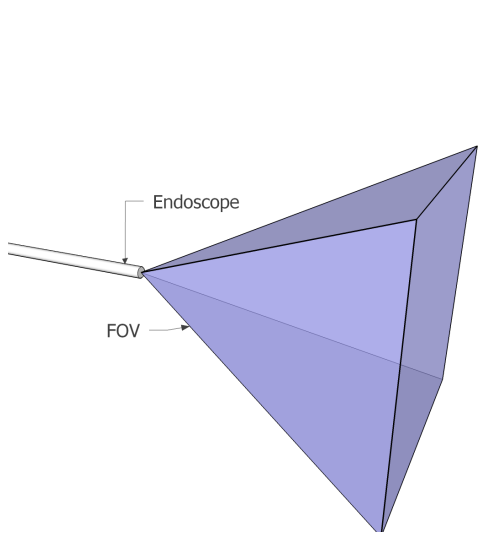

(a)

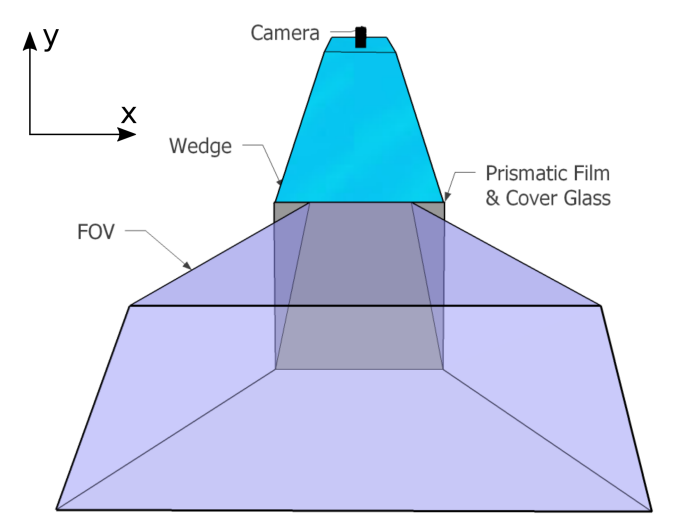

(c)

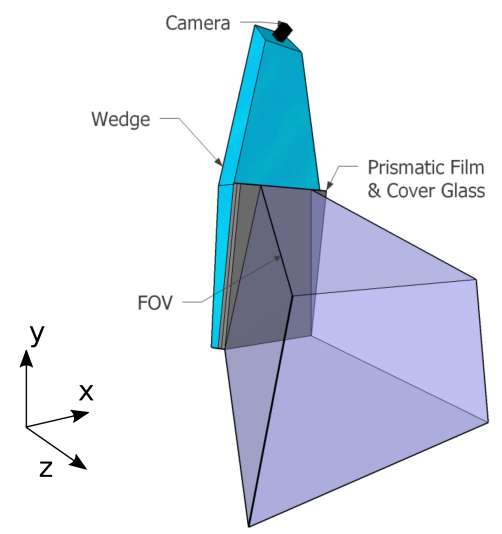

(b)

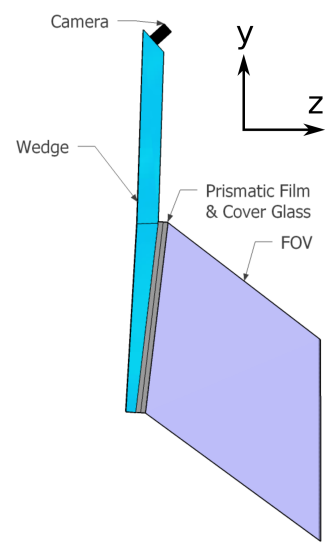

(d)

Fig. 4. (a) Typical FOV of an endoscope or a regular camera. (b) FOV of the proposed device. (c,d) Front and side views of $b$. 

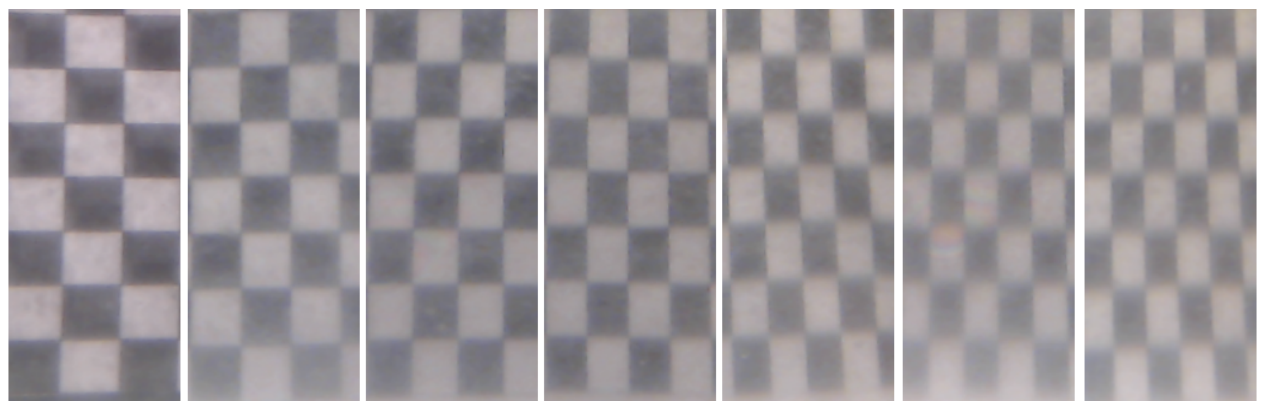

Fig. 5. The effect of the $1 D$ perspective on a checkerboard at (from left to right) $0 \mathrm{~cm}, 5 \mathrm{~cm}, 10 \mathrm{~cm}, 15 \mathrm{~cm}, 20 \mathrm{~cm}$, $25 \mathrm{~cm}, 30 \mathrm{~cm}$ away from the wedge surface

presented in this paper, we used the Logitech C310 webcam, however, many other cameras could be used as well. For example, the higher resolution Logitech C922 webcam produced even better results.

\subsection{Illumination}

The simplest way of illuminating a surface under inspection is to shine light from a point next to the camera. The surface will then receive uniform illumination whereas lamps placed round the sides of the wedge would illuminate the edges of the scene more than the center. Experiments in the laboratory showed, however, that a fraction of the illumination reflects off the prism facets back towards the camera and dazzles the camera along a vertical line. A simple yet effective way to solve this is to rotate the prismatic film by approximately $15^{\circ}$ about the surface normal as shown in Figure 6 . The reflected light is then at $30^{\circ}$ to the wedge axis so that the dazzling line moves off to one side and therefore out of the way of the surface under inspection.
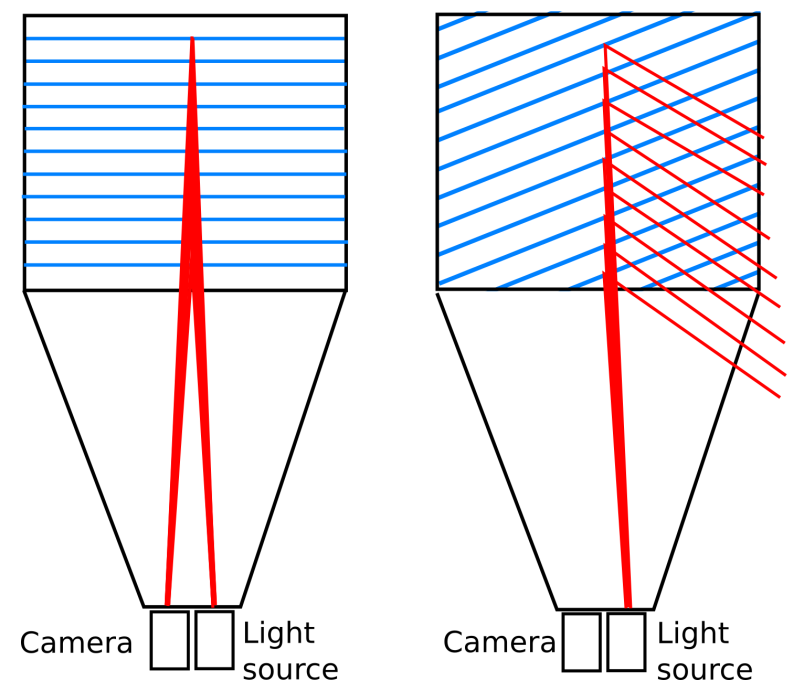

Fig. 6. By rotating the prismatic film, the camera is no longer dazzled by the specular reflection of the light source.

ACM J. Comput. Cult. Herit., Vol. 12, No. 2, Article . Publication date: June 2019. 


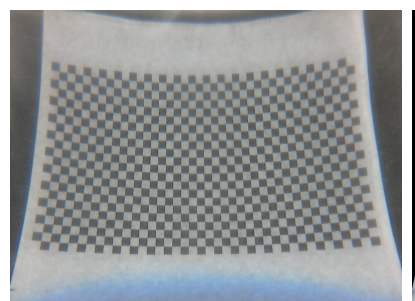

(a)

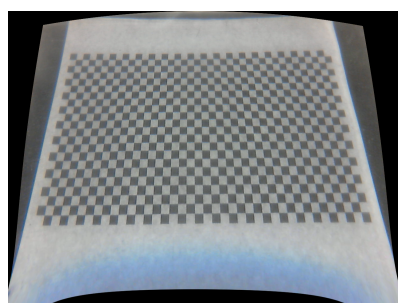

(b)

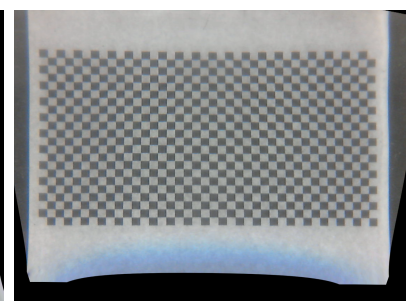

(c)

Fig. 7. Software correction: (a) Raw image; (b) pincushion distortion correction; (c) pincushion and keystone distortion correction.

We just described a way to illuminate from the device itself. However, in practice, most of the time we relied on sunlight to illuminate areas under inspection. We could also use mirrors to redirect natural light into trenches but this would probably require the trenches to be larger.

\subsection{Software and calibration}

The acquired raw images of the wedge guide cannot be used directly as there are strong distortions that arise because the wedge and the camera behave somewhat like a camera and a Fresnel lens in an off-axis configuration. Consequently, we propose to correct the acquired images in real-time with image processing, just as is done in other computational imaging systems. We developed a software solution in $\mathrm{C}++$ with the OpenCV computer vision library that processes the stream of the camera in real-time. Hence, we can directly visualize and record the corrected acquired images.

The distortions consist of a strong pincushion distortion as well as a keystone distortion. For the calibration, in a pre-processing step, we use a series of images of a checkerboard in order to calculate the correction for the pincushion distortion (Figure 7(b)). Then, we manually select the corners of the checkerboard in order to compute the homography matrix for the keystone correction (Figure 7(c)). After this calibration, the corrections are applied in real-time for any frame of the video stream in order to provide the archaeologists with the correct visualization.

\section{CASE STUDY}

We tested our wedge camera at two archaeological sites in Egypt near Luxor, namely Deir El Medina and Medamoud. We identified three concrete applications where wedge cameras can aid inspection in confined spaces, and we present results and what we learnt in the following.

First, wedge cameras can image the underground through slim trenches (Section 4.1), second, underwater surveys can be done by imaging flooded trenches (Section 4.2), and third, areas in confined spaces such as structural crevices or between stones in a wall can be imaged (Section 4.3).

\subsection{Trial trenches}

Trial trenches are usually dug before a full excavation is launched so that archaeologists can look at layers of soil beneath the surface and see if there are remains dating from layers corresponding to the epoch of interest. The trenches must be big enough for someone to get into, i.e. about 1 meter wide, typically two metres long and from one to several metres deep. Digging such trenches is time consuming, and, moreover, 
it is moderately invasive and this will bear on the decision of local authorities to grant permission to dig. With the wedge, it is possible to get some visual information from a trench and be much less invasive.

Digging a deep trench that is only 5 to 10 centimetres wide proved difficult in European clay soil and would no doubt be difficult in dry sand. However, on the site where the wedge cameras were tested in Medamoud, the soil is less prone to flow or crumble and a simple trenching tool was enough to dig trenches to arm's length as recorded in Table 1. Rays undergo approximately 20 internal reflections within the wedge guide so its surfaces must be especially smooth (roughness average of $1 \mathrm{~nm}$ ) and clean. It might therefore seem optimistic to have placed the wedge in trenches swirling with grit and dust but although there was some degradation of the image, it was not drastic.

\begin{tabular}{|c|c|c|c|c|c|}
\hline Trench & Length at the surface & Width at the surface & Length at the bottom & Width at the bottom & Depth \\
\hline 1 & $75 \mathrm{~cm}$ & $14 \mathrm{~cm}$ & $28 \mathrm{~cm}$ & $11 \mathrm{~cm}$ & $70 \mathrm{~cm}$ \\
\hline 2 & $45 \mathrm{~cm}$ & $18 \mathrm{~cm}$ & $26 \mathrm{~cm}$ & $7 \mathrm{~cm}$ & $70 \mathrm{~cm}$ \\
\hline 3 & $55 \mathrm{~cm}$ & $20 \mathrm{~cm}$ & $25 \mathrm{~cm}$ & $8 \mathrm{~cm}$ & $63 \mathrm{~cm}$ \\
\hline
\end{tabular}

Table 1. Dimensions of the trenches.

The wedge camera was inserted and it quickly became apparent that the soil is much darker than one might expect. Illumination that was perfectly adequate in the laboratory gave no useful image in the trench and the best images were obtained by allowing sunlight to leak down into the trench. Time and again, the authors found themselves experimenting with different ways of illuminating the scene and perhaps this should not be too surprising because free-space photography requires much the same care with illumination.

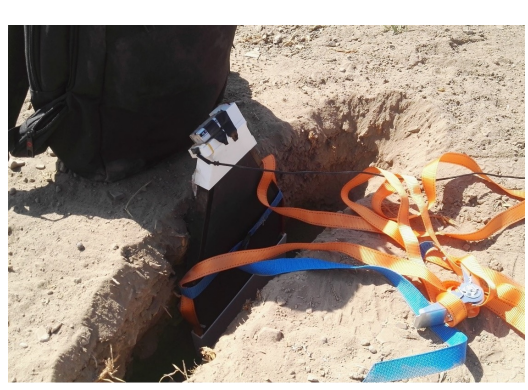

(a)

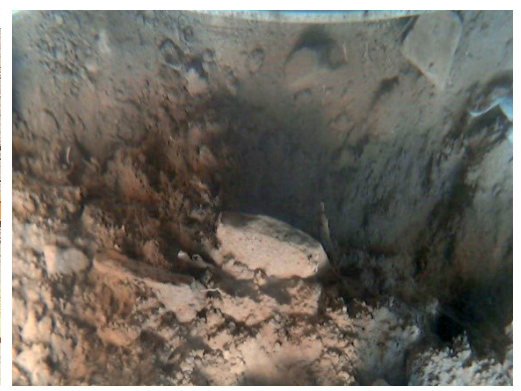

(b)

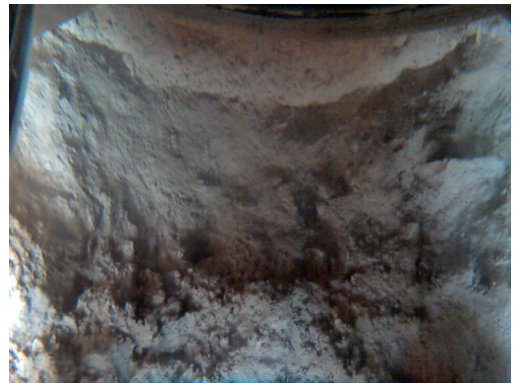

(c)

Fig. 8. (a) The wedge down a slim trench; (b) and (c) Images of walls of trenches acquired from the device.

Once the illumination had been optimized, images were produced that were much the same as would be obtained through free space. However, archaeologists commented that the differences in reflectivity and texture that delineate different strata are difficult to see unless the sides of the trench walls are carved flat and smooth. Giving walls such a finish is not so difficult in a wide trench but is more challenging when the wall cannot be inspected at the same time that it is being perfected. It might be possible to create narrow trenches with the surface finish needed for useful inspection, for example by using a plough-share.

The authors note that whereas permission was given for conventional trial trenches to be dug only in specific locations on site, trenches adequate for the wedge camera were sufficiently narrow that we were 
allowed to dig them anywhere on site. Moreover, it happens that a ground survey is necessary in areas that are constrained by a structure, so the wedge camera might find many applications for observing the underground in specific conditions.

\subsection{Underwater surveys}

It is not uncommon, particularly in Egypt, for the water table to be high so that traditional surveys cannot be performed because they would rapidly be flooded. Our wedge camera system can address this issue as it can work underwater.

The wedge principle as described in Section 2 is based on the value of the critical angle, which is the limit between total internal reflection and refraction. This value depends on the indices of refraction $n$ of both optical media: the acrylic that makes the wedge $(n=1.49)$ and air $(n=1.00)$ which is the exit medium. The wedge that we use is designed to work in air, so if the wedge surface is in direct optical contact with the water $(n=1.33)$, the critical angle is reduced, and the rays travel less far within the wedge (Figure 9(a)). This phenomenon is clearly unwanted as the image would be squeezed in one direction.

It follows that an air interface is desirable between the wedge and the water but the water itself will refract light the same way the prismatic film described above does, so the prismatic film may not be needed if the air interface is provided (Figure 9(b)).

In our test, however, we used a flexible transparent bag and could not ensure the air interface when put inside water. We therefore kept the prismatic film so that the space between prisms acts as an air interface, and we used the system depicted in Figure 9(c).

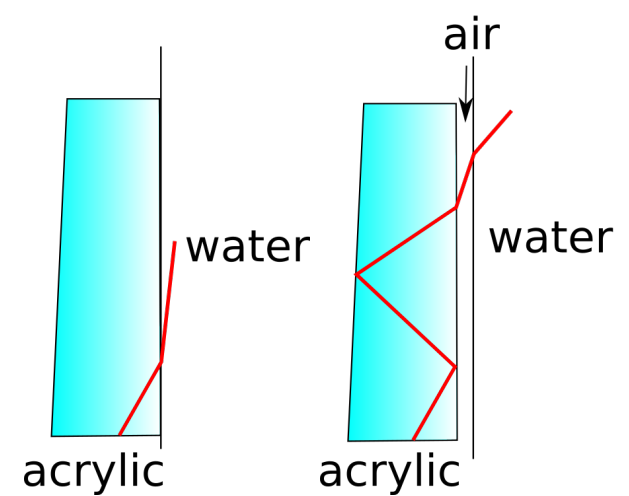

(a) (b)

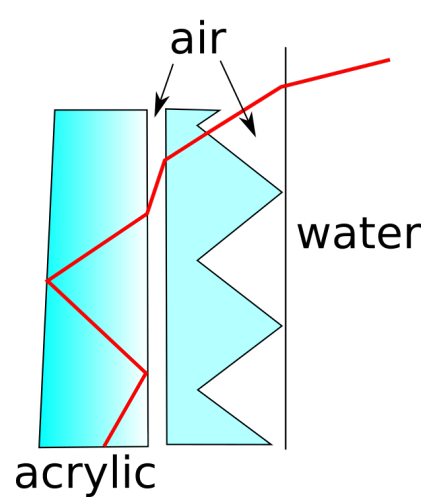

(c)

Fig. 9. a) The wedge directly in water would make the rays experience less bounces. b) When the air interface is ensured, the prismatic film may not be needed to redirect light rays. c) Our test setup with the prismatic film and a transparent plastic bag, with air interfaces.

A $10 \mathrm{~cm}$ wide trench was dug beneath the water table (Figure 10(a)) and then left overnight for the mud to settle. The wedge camera was wrapped in the flexible transparent sheet and lowered into the trench. The captured images were adequate even if rapidly degraded by the upwell of silt, but as in Section 4.1, it was difficult to see any changes in reflectivity or texture, perhaps because experts are more used to work in open air. Some tests were also performed to inspect the walls of a flooded chamber 
(Figure 10(b)). We could achieve an image quality that was as good as if the pictures were taken in open air.

We therefore think that our wedge light guide system may also find application in underwater archaeology.

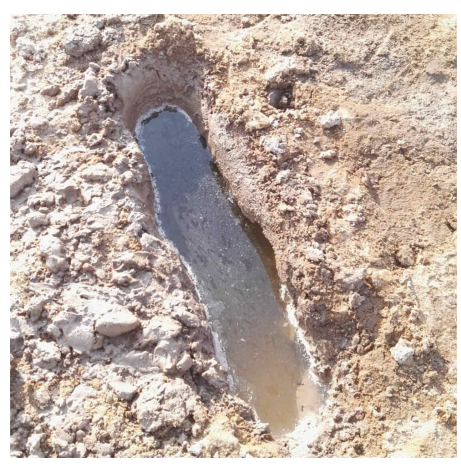

(a)

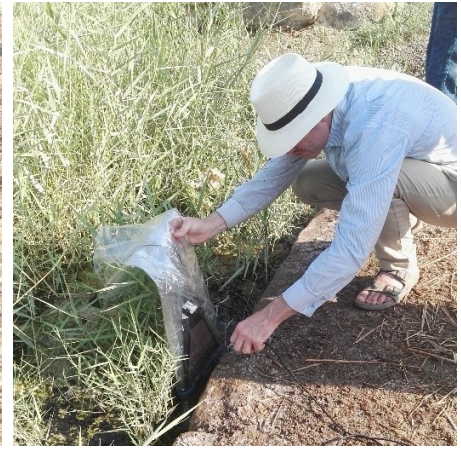

(b)

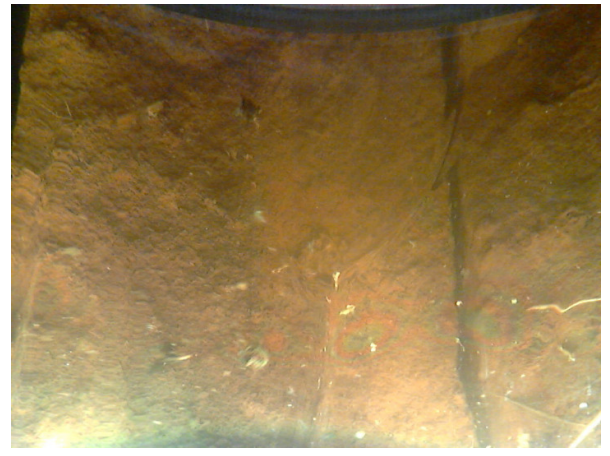

(c)

Fig. 10. (a) An overflooded trench where the wedge can be used; (b) a wedge being used for observing the side of a flooded area; (c) image acquired underwater.

\subsection{Structural crevices}

Crevices are common in ancient structures where a wall is rarely so smooth that there is no gap between their stones. The interest in these gaps arises because it was not unusual for temples to be disassembled and rebuilt with the stones of previous temples built on the same site. Decorations from these earlier temples were often unwanted and so the decorated surfaces were placed out of view adjacent to other stones. These decorations cannot be observed without considerable disruption (such as removing temple stones) unless a wedge is used. Figure 11 shows situations where the wedge camera can be useful to inspect these constrained areas. The device can notably help a lot in the field of epigraphy, where writings are not always easily accessible. As an example, Figure 12 shows the uncorrected and corrected image of a hieroglyph taken from the crevice of Figure 11(a) with the wedge camera. The wedge was placed at a few centimeters away from the wall yet we could achieve a large imaging area with good image quality.

Most crevices between the stones of a temple do not of course contain images on the opposing surfaces. Proving that they do not is useful in itself and the wedge camera was used to do this in several locations such as that of Figure 11(c). Once again, experimenting in order to optimize illumination was important in order to be sure that there really were no images to be seen. The crevice of Figure 11(b) was unfortunately slightly too narrow for the wedge used in these trials. Archaeologists suggested that a smaller, phone-sized wedge could be useful for quick inspection on more potential places. They emphasized that it is not only inscriptions that interest them, for example much can be learned about techniques of construction from marks left by the devices used to move the stones and by the procedures used to align them. There was a degree of enthusiasm that the richness of information given by the device might lead to unforeseen discoveries. 


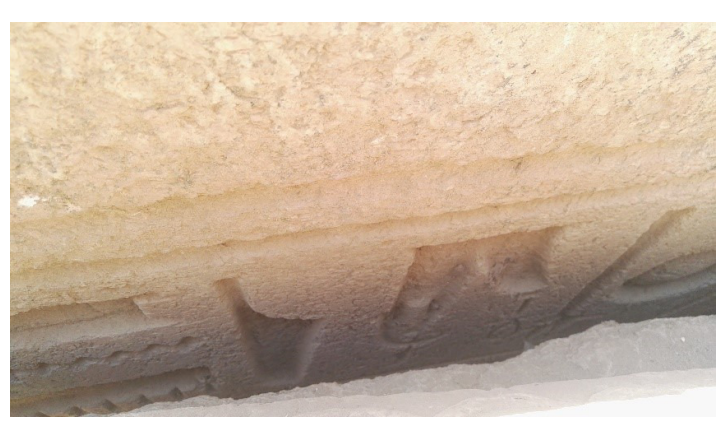

(a)

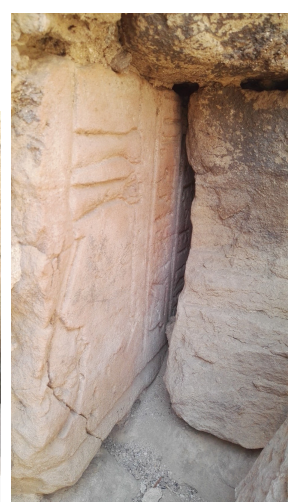

(b)

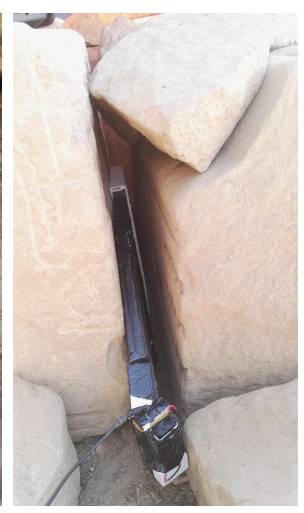

(c)

Fig. 11. Typical examples of wall crevices that could be inspected with a wedge.

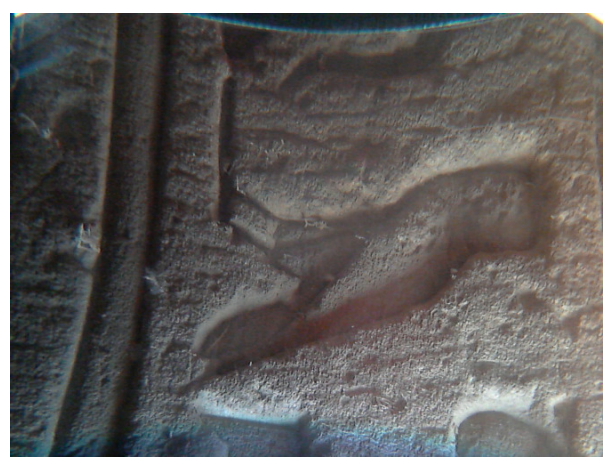

(a)

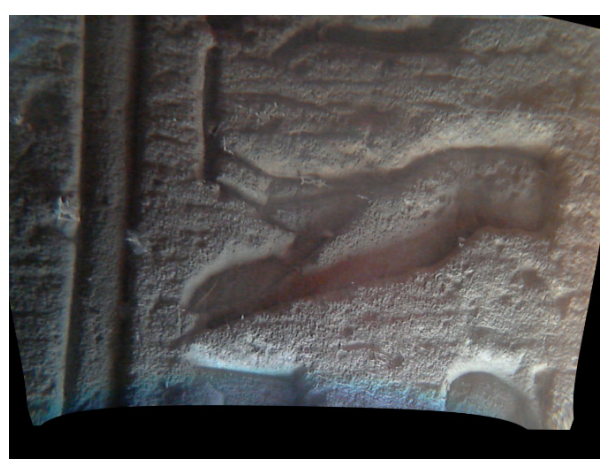

(b)

Fig. 12. (a) Raw image of a hieroglyph; (b) image corrected from distortion.

\section{FUTURE WORK}

This project and our device is opening new directions of research, development and applications. In this section, we explain the prospective work and this could give an idea of its full potential.

Firstly, the image quality can be degraded when the device is used onsite, because the wedge guide is very sensitive to dust and scratches. When experimenting at Medamoud, we observed that the sand that was in contact with our glass protective layer inevitably degraded the image quality. The images presented here were a bit modified to improve the contrast ratio and brightness. It could be interesting to develop new filters to offer archaeologists an improved experience with high quality images. Moreover, the calibration process could be improved by calibrating the color correction.

We listened to the remarks expressed by archaeologists and we will test a smaller wedge that could fit into more crevices, for example with an A5 active area and a a thickness of $10 \mathrm{~mm}$. Of course, the downside is that the size of the region of interest would be smaller. Indeed, the image size is currently limited to the size of the device but it could be interesting to stich images together [8] in order to provide the full profile of a trench or a wall, and/or to increase their resolution, using super-resolution techniques [13]. This implies some software and hardware challenges such that ensuring that the illumination is 
uniform within the frame and coherent between several frames. Illumination may also be easily improved with high-powered LEDs.

Finally, it would be interesting to investigate the potential of the device in terms of 3D reconstruction. It is possible to put several cameras at the end of the wedge guide, so that different viewpoints of the same scene can be taken simultaneously. This can be used to extract depth information from parallax and generate depth maps of objects under inspection, as with any stereo camera. Our first tests are encouraging, however, some work still needs to be to done to fully evaluate the accuracy of such a method. A projector might be associated with one or several cameras to perform 3D reconstruction from structured illumination [5]. Moreover, as images at different angles can be taken either simultaneously or by moving the device, we think it should be possible to reconstruct a 3D mesh from them by photogrammetry. This is not direct though: our device calibration is not standard, stable illumination of the scene is hard to achieve, and tilting the device in a trench can be hard so that only few angles might be accessible. To this end, using a better camera would be mandatory, with a better resolution and probably a global shutter because it may be difficult to hold the device still. Changes in the core of photogrammetry algorithms may also be necessary, notably adapting the calibration process and changing the camera model to a "pusbroom-like" model. Looking further into the future, it might be useful to combine the wedge light guide with a close range laser scanner. Indeed, the laser light could propagate in the wedge from a laser source to the scene back to a detector to evaluate depth information with time of flight techniques [4].

\section{CONCLUSIONS}

In this paper, we presented a novel hardware optical device, a wedge camera, associated with software processing that can be used in archaeology to get images where traditional cameras fail. We tested and discussed three concrete applications.

Before using our wedge camera, specialists in the digging of trial trenches were doubtful that our light guide would bring them much benefit because of the lack of pure visible information from the ground, and they were reluctant to lose the ability to touch and interact with the sides of a trial trench. However, after testing, they agreed that the wedge could help inspecting areas where traditional surveys do not fit. They also mentioned that it might be easier to get authorization for surveys when conducted with a wedge because it keeps disruption to a minimum.

The underwater application interested them because it means that one could observe trenches that are deeper than usual, even below the water line. Wedges might be used for the inspection of lakes and also in submarine archaeology.

There was more interest, even enthusiasm, in using the wedge camera to look into crevices. We have concentrated on the use of the wedge in Egyptology because of their need to inspect temples, but it can likely be used in many other applications such as in the archaeology of buildings, the exploration of caverns, among others, even beyond archaelogy.

\section{ACKNOWLEDGMENTS}

The authors would like to thank the Institut Français d'Archéologie Orientale for a grant and Microsoft Corporation for their support. Also Cédric Gobeil, leader of the mission at Deir El-Medina, Félix Relats, leader of the mission at Medamoud and Ahmed Muhammad Sayed el Naseh, Ministry of Antiquities. We thank the anonymous reviewers for their interesting remarks.

\section{REFERENCES}

[1] Ronald G. Beckett. 2015. Application and Limitations of Endoscopy in Anthropological and Archaeological Research. The Anatomical Record 298, 6 (2015), 1125-1134. https://doi.org/10.1002/ar.23145

ACM J. Comput. Cult. Herit., Vol. 12, No. 2, Article . Publication date: June 2019. 
[2] Leandro Bornaz and Fulvio Rinaudo. 2004. Terrestrial laser scanner data processing. In XXth ISPRS Congress Istanbul.

[3] Sophie Boual, Timothy Large, Mark Buckingham, Adrian Travis, and Simon Munford. 2006. 72.3: Wedge Displays as Cameras. SID Symposium Digest of Technical Papers 37, 1 (2006), 1999-2002. https://doi.org/10.1889/1.2433445

[4] Carlo Bianchini Gabriele Guidi. 2007. TOF laser scanner characterization for low-range applications. (2007), 6491 6491 - 11 pages. https://doi.org/10.1117/12.705288

[5] Guido Gerig. 2012. Structured Lighting. (2012).

[6] Rajiv Gupta and Richard I. Hartley. 1997. Linear Pushbroom Cameras. IEEE Trans. Pattern Anal. Mach. Intell. 19, 9 (Sept. 1997), 963-975. https://doi.org/10.1109/34.615446

[7] Klaus Hanke and Pierre Grussenmeyer. 2002. Architectural Photogrammetry: basic theory, procedures, tools. In ISPRS Commission, Vol. 5. 1-2.

[8] Rick Szeliski. 2004. Image Alignment and Stitching: A Tutorial. Technical Report. 89 pages. https://www.microsoft. com/en-us/research/publication/image-alignment-and-stitching-a-tutorial/

[9] A.R.L. Travis. 2003. Flat-panel display using tapered waveguide. (Dec. 18 2003). Patent WO/2003/013151.

[10] ARL Travis, F Payne, F Zhong, and JR Moore. 2000. Flat panel display using projection within a wedge-shaped waveguide. In Proceedings of the 20th International Display Research Conference, Vol. 2000. Society for Information Display (SID), 292-295.

[11] Adrian RL Travis, Tim Large, Neil Emerton, Zhaoming Zhu, and Steven Bathiche. 2010. Image capture via a wedge light-guide with no margins. Optics express 18, 8 (2010), 8453-8458.

[12] Adrian RL Travis, Timothy A Large, Neil Emerton, and Steven N Bathiche. 2013. Wedge optics in flat panel displays. Proc. IEEE 101, 1 (2013), 45-60.

[13] Linwei Yue, Huanfeng Shen, Jie Li, Qiangqiang Yuan, Hongyan Zhang, and Liangpei Zhang. 2016. Image superresolution: The techniques, applications, and future. Signal Processing 128 (2016), 389 - 408. https://doi.org/10.1016/ j.sigpro.2016.05.002

accepted July 2018

ACM J. Comput. Cult. Herit., Vol. 12, No. 2, Article . Publication date: June 2019. 